\title{
Thermodynamic analysis on the cononsolvency of poly (vinyl alcohol) in water-DMSO mixtures through the ternary interaction parameter
}

\author{
Tai-Horng Young*, Wen-Yuan Chuang \\ Institute of Biomedical Engineering, College of Medicine and College of Engineering, \\ National Taiwan University, Taipei 10016, Taiwan, ROC
}

Received 15 April 2002; accepted 9 September 2002

\begin{abstract}
Isothermal phase diagrams for the semicrystalline poly (vinyl alcohol) (PVA) in solutions composed of water and dimethylsulfoxide (DMSO) was studied at $25^{\circ} \mathrm{C}$. From the observed phase behavior, PVA was soluble in either water or DMSO individually but crystallization-induced gelation and liquid-liquid demixing were observed in water-DMSO mixtures. Flory-Huggins formalism including three binary interaction parameters and one ternary interaction parameter was used to study the phenomenon of the cononsolvency, i.e. the formation of nonsolvents by mixing two solvents. The equilibrium crystallization line in the DMSO-rich region and the total calculated binodals agreed well with the measured results when a composition-dependent ternary interaction parameter was included into calculations. In contrast, calculations yielded crystallization-induced gelation in the water-rich region, but experiments indicated that PVA remained well dissolved even 1 year after preparation. The discrepancy was explained by the temperature-induced changes in the relative interaction between water and PVA. In addition, the role of the ternary interaction parameter in the cononsolvent ternary polymer systems was discussed. It was found the contribution of the ternary interaction parameter in the cononsolvent system under study is to decline the degree of the cononsolvency. The driving force for cononsolvency is the strong interaction between water and DMSO to form the stable DMSO hydrate to exclude PVA segments in the vicinity of the hydrate.
\end{abstract}

(C) 2002 Elsevier Science B.V. All rights reserved.

Keywords: Phase behavior; Cononsolvency; Ternary interaction parameter

\section{Introduction}

Poly (vinyl alcohol) (PVA) is nowadays widely used in scientific and technological fields, due to their good chemical stability, thermal stability and high water permeability [1]. Recently, many studies have been carried out on PVA gels from solutions of mixtures

\footnotetext{
* Corresponding author. Tel.: +886-2-23123456x1455; fax: +886-2-23940049.

E-mail address: thyoung@ha.mc.ntu.edu.tw (T.-H. Young).
}

of water and dimethylsulfoxide (DMSO) [2-5]. Cha et al. succeeded in producing PVA fibers having highly drawability by gel spinning of PVA from spinning dope with the PVA concentration of $6 \mathrm{wt} . \%$ consisting of DMSO-water mixtures [2]. Ohkura et al. reported the sol-gel behavior of the PVA gels in a mixture of DMSO and water at quenching temperatures between -60 and $80^{\circ} \mathrm{C}$ [3]. It was pointed out that the gelation behavior from this mixture depends on the competition between crystallization and liquid-liquid phase separation. Kanaya et al. suggested from wide- and 
small-angle neutron scattering measurements that the cross-linking points of the PVA gels are crystallites [4]. Yamaura et al. studied the gel structure from the flow temperature of gels by repeated freezing/thawing cycles $\left(-78\right.$ to $\left.20^{\circ} \mathrm{C}\right)$ with water/DMSO $=90 / 10$ and $50 / 50$ [5]. If all the free water is assumed to have transuded by syneresis, the residual bound water is about six water molecules per one vinyl alcohol monomer unit.

It is well known that a phase diagram can provide important information in various applications such as the various phase transitions that are responsible for the preparation of membranes by immersion of a polymer solution in a coagulation bath. Although the phase diagram for the system water-DMSO has been studied [6], there has been no investigation in constructing a complete phase diagram of PVA in water-DMSO mixtures as far as we know. For comparison with the present study, the phase diagram of the ternary system water (nonsolvent), DMSO (solvent), poly (ethylene-co-vinyl alcohol) (EVAL) at $25^{\circ} \mathrm{C}$ described previously [7] is shown in Fig. 1. In this typical nonsolvent-solvent polymer system, the crystallization-induced gelation and bin- odal boundaries are near the solvent apex and the latter is located inside the former. Such a typical phase behavior is quite distinct from that of PVA in the same binary solvents, as indicated in Fig. 2. Two crystallization-induced gelation boundaries and two binodal boundaries were constructed by experimental measurements. PVA was soluble in either water or DMSO independently, but phase separation occurred at mixtures of water and DMSO. Due to the reduction of the solvent power at some composition of the mixed solvent, the water-DMSO mixture is termed cononsolvent [8] for PVA, hereinafter. In the field of ternary polymer systems the so-called cononsolvency is an interesting phenomenon that has not been subjected to extensive investigation from the thermodynamic point of view [8-10]. Thus, the objective of this paper was to investigate the complete phase diagram on the basis of a suitable thermodynamic model. The phase equilibrium of a ternary polymer system has been derived from Flory-Huggins thermodynamics based on the binary interaction parameters [11]. In this paper we used the ternary parameter, $\chi_{\mathrm{T}}$ proposed by Pouchly et al. [12] to improve the agreement between experimental and theoretical data. We found

\section{EVAL}

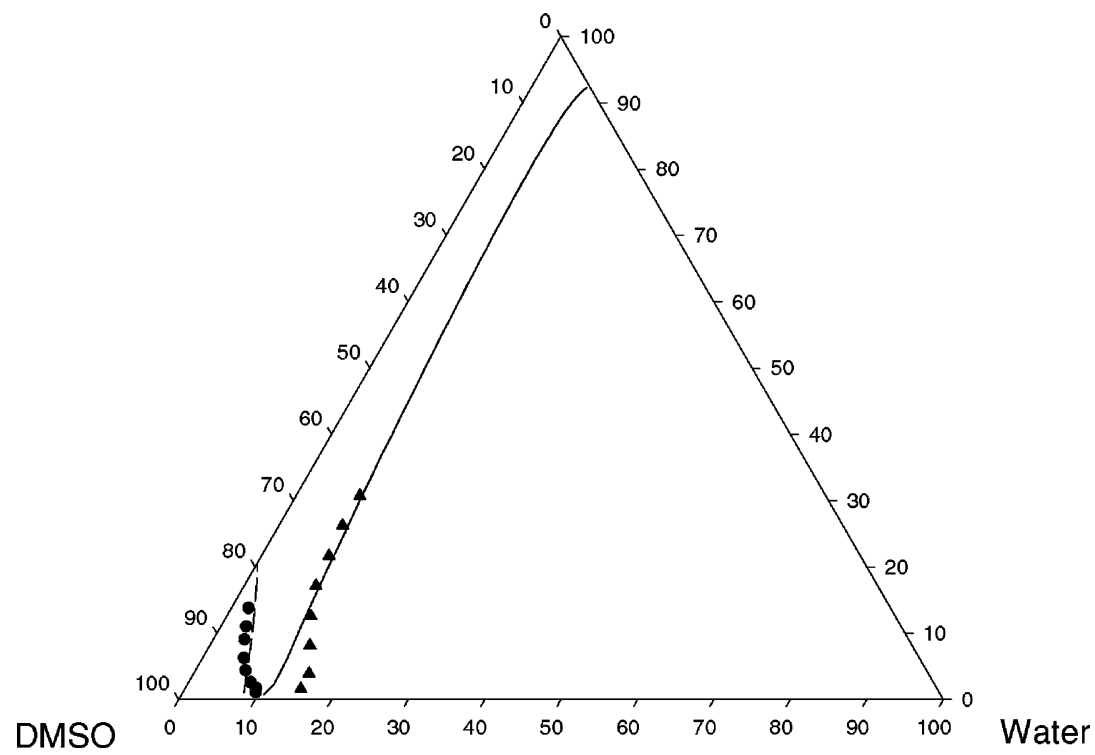

Fig. 1. Phase diagram of water-DMSO-EVAL at $25^{\circ} \mathrm{C}$ : $(\mathbf{O})$ measured gel points; $(\mathbf{\Delta})$ measured binodal points; (- - -) computed crystallization equilibrium line; (-) computed binodal equilibrium line. 


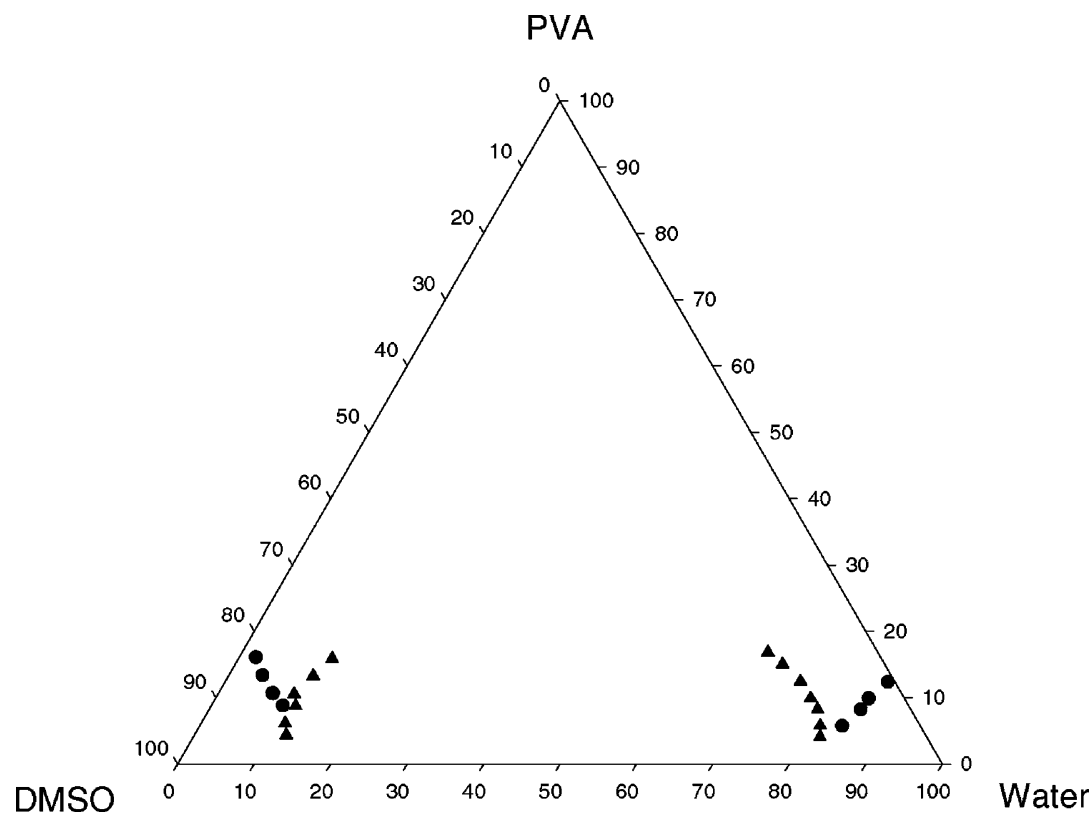

Fig. 2. Phase diagrams of water-DMSO-PVA at $25^{\circ} \mathrm{C}$. The data points, denoted by filled triangles $(\mathbf{\Delta})$ and circles $(\boldsymbol{O})$, represent the composition of the binodal- and crystallization-induced gelation boundaries, respectively.

that only binary interaction parameters provided an inadequate description of the phase behavior of PVA in water-DMSO mixtures. When a ternary interaction parameter was considered, an improvement could be obtained and unknown phase boundaries that could not be obtained experimentally could be predicted, except the discrepancy in the water-rich region. The state of water in water-swollen polymer has been studied by numerous researchers [13-15]. Hodge et al. proposed that water molecules usually exist in two or three separate states within the PVA: nonfreezing, freezable bound, and free water [13]. The nonfreezing water by attaching themselves to hydroxyl groups on the PVA in the first stage of water up-taking is responsible for the destruction of PVA crystalline structure. Above a characteristic level of water for each hydroxyl group, a number of freezable bound water molecules aggregate around the polar site of the polymer with sufficient structural order but greatly influenced by interactions with polymers. Water does not exist within the PVA as freezable water until the hydrogen bonding in the PVA has been disrupted by the nonfreezing and freezable bound water. Hence, the effects of water state within the PVA and its interaction with PVA on the phase behavior were discussed. Finally, the roles of the ternary and binary interaction parameters in the phenomenon of cononsolvency were also discussed.

\section{Theory}

Flory-Huggins theory was used to describe the phase behavior for ternary polymer solutions [11], in which a ternary interaction parameter, $\chi_{\mathrm{T}}$, was included $[12,16]$. The Gibbs free energy of mixing $\left(\Delta G_{\mathrm{m}}\right)$ for the polymer solution is

$$
\begin{aligned}
\Delta G_{\mathrm{m}}= & R T\left(n_{1} \ln \phi_{1}+n_{2} \ln \phi_{2}+n_{3} \ln \phi_{3}+\chi_{12} n_{1} \phi_{2}\right. \\
& \left.+\chi_{13} n_{1} \phi_{3}+\chi_{23} n_{2} \phi_{3}+\chi_{\mathrm{T}} n_{1} \phi_{2} \phi_{3}\right)
\end{aligned}
$$

where $n_{i}$ and $\phi_{i}$ are numbers of moles and volume fraction of component $i(i=1$ : water; $i=2$ : DMSO; $i=3$ : PVA). $\chi_{i j}$ is a binary interaction parameter between component $i$ and component $j$. Differentiating Eq. (1) with respect to $n_{i}$, the chemical potential, 
$\Delta \mu_{i}\left(\mu_{i}-\mu_{i}^{0}\right)$ of the three components is given:

$$
\begin{aligned}
\frac{\Delta \mu_{1}}{R T}= & \ln \phi_{1}+1-\phi_{1}-\frac{V_{1}}{V_{2}} \phi_{2}-\frac{V_{1}}{V_{3}} \phi_{3} \\
& +\left(\phi_{2} \chi_{12}+\phi_{3} \chi_{13}\right)\left(\phi_{2}+\phi_{3}\right)-\frac{V_{1}}{V_{2}} \phi_{2} \phi_{3} \chi_{23} \\
& -h_{1} h_{2} \phi_{2} \frac{\mathrm{d} \chi_{12}}{\mathrm{~d} h_{2}}-\phi_{1} \phi_{2} \phi_{3} \frac{\partial \chi_{13}}{\partial \phi_{2}}-\phi_{1} \phi_{3}^{2} \frac{\partial \chi_{13}}{\partial \phi_{2}} \\
& -\phi_{1} \phi_{3}^{2} \frac{\partial \chi_{13}}{\partial \phi_{3}}-\frac{V_{1}}{V_{2}} \phi_{2}^{2} \phi_{3} \frac{\partial \chi_{23}}{\partial \phi_{2}}-\frac{V_{1}}{V_{2}} \phi_{2} \phi_{3}^{2} \frac{\partial \chi_{23}}{\partial \phi_{3}} \\
& -\phi_{1} \phi_{2}^{2} \phi_{3} \frac{\partial \chi_{\mathrm{T}}}{\partial \phi_{2}}-\phi_{1} \phi_{2} \phi_{3}^{2} \frac{\partial \chi_{\mathrm{T}}}{\partial \phi_{3}} \\
& +\chi_{\mathrm{T}} \phi_{2} \phi_{3}\left(1-2 \phi_{1}\right)
\end{aligned}
$$

$$
\begin{aligned}
\frac{\Delta \mu_{2}}{R T}= & \ln \phi_{2}+1-\phi_{2}-\frac{V_{2}}{V_{1}} \phi_{1}-\frac{V_{2}}{V_{3}} \phi_{3} \\
& +\left(\frac{V_{2}}{V_{1}} \phi_{1} \chi_{12}+\phi_{3} \chi_{23}\right)\left(\phi_{1}+\phi_{3}\right) \\
& -\frac{V_{2}}{V_{1}} \phi_{1} \phi_{3} \chi_{13}+\frac{V_{2}}{V_{1}} h_{1} h_{2} \phi_{1} \frac{\mathrm{d} \chi_{12}}{\mathrm{~d} h_{2}} \\
& +\frac{V_{2}}{V_{1}} \phi_{1} \phi_{3}\left(\phi_{1}+\phi_{3}\right) \frac{\partial \chi_{13}}{\partial \phi_{2}}-\frac{V_{2}}{V_{1}} \phi_{1} \phi_{3}^{2} \frac{\partial \chi_{13}}{\partial \phi_{3}} \\
& +\phi_{2} \phi_{3}\left(\phi_{1}+\phi_{3}\right) \frac{\partial \chi_{23}}{\partial \phi_{2}}-\phi_{2} \phi_{3}^{2} \frac{\partial \chi_{23}}{\partial \phi_{3}} \\
& +\frac{V_{2}}{V_{1}} \phi_{1} \phi_{2} \phi_{3}\left(\phi_{1}+\phi_{3}\right) \frac{\partial \chi_{\mathrm{T}}}{\partial \phi_{2}} \\
& -\frac{V_{2}}{V_{1}} \phi_{1} \phi_{2} \phi_{3}^{2} \frac{\partial \chi_{\mathrm{T}}}{\partial \phi_{3}}+\frac{V_{2}}{V_{1}} \chi_{\mathrm{T}} \phi_{1} \phi_{3}\left(1-2 \phi_{2}\right)
\end{aligned}
$$

$$
\begin{aligned}
\frac{\Delta \mu_{3}}{R T}= & \ln \phi_{3}+1-\phi_{3}-\frac{V_{3}}{V_{1}} \phi_{1}-\frac{V_{3}}{V_{2}} \phi_{2} \\
& +\left(\frac{V_{3}}{V_{1}} \phi_{1} \chi_{13}+\frac{V_{3}}{V_{2}} \phi_{2} \chi_{23}\right)\left(\phi_{1}+\phi_{2}\right) \\
& -\frac{V_{3}}{V_{1}} \phi_{1} \phi_{2} \chi_{12}-\frac{V_{3}}{V_{1}} \phi_{1} \phi_{2} \phi_{3} \frac{\partial \chi_{13}}{\partial \phi_{2}} \\
& -\frac{V_{3}}{V_{2}} \phi_{2}^{2} \phi_{3} \frac{\partial \chi_{23}}{\partial \phi_{2}}+\phi_{3}\left(\phi_{1}+\phi_{2}\right) \\
& \times\left[\frac{V_{3}}{V_{1}} \phi_{1} \frac{\partial \chi_{13}}{\partial \phi_{3}}+\frac{V_{3}}{V_{2}} \phi_{2} \frac{\partial \chi_{23}}{\partial \phi_{3}}\right] \\
& -\frac{V_{3}}{V_{1}} \phi_{1} \phi_{2}^{2} \phi_{3} \frac{\partial \chi_{\mathrm{T}}}{\partial \phi_{2}}+\frac{V_{3}}{V_{1}} \phi_{1} \phi_{2} \phi_{3}\left(\phi_{1}+\phi_{2}\right) \frac{\partial \chi_{\mathrm{T}}}{\partial \phi_{3}} \\
& +\frac{V_{3}}{V_{1}} \chi_{\mathrm{T}} \phi_{1} \phi_{2}\left(1-2 \phi_{3}\right)
\end{aligned}
$$

where $\mu_{i}^{0}$ is the chemical potential at standard state, $V_{i}$ is the molar volume, $h_{1}=\phi_{1} /\left(\phi_{1}+\phi_{2}\right)$ and $h_{2}=$ $\phi_{2} /\left(\phi_{1}+\phi_{2}\right)$.

At liquid-liquid phase equilibrium at a specified temperature and pressure, the chemical potential of each component between two phases $(\alpha$ and $\beta$ ) is equal:

$\mu_{i}^{\alpha}=\mu_{i}^{\beta} \quad(i=1,2,3)$

where $\mu_{i}^{\alpha}$ and $\mu_{i}^{\beta}$ are the chemical potentials of component $i$ in phases $\alpha$ and $\beta$, respectively.

At the crystallization equilibrium, the modified Flory-Huggins expression for the chemical potential between the liquid phase and the polymer crystal in a ternary system derived by Cheng et al. [17] was followed:

$$
\begin{gathered}
\frac{1}{R T}\left[\Delta H_{\mathrm{f}}\left(1-\frac{T}{T_{\mathrm{m}}}\right)\right]+\frac{V_{\mathrm{u}}}{V_{3}} \ln \phi_{3}+\frac{V_{\mathrm{u}}}{V_{3}}\left(1-\phi_{3}\right) \\
-\frac{V_{\mathrm{u}}}{V_{1}} \phi_{1}-\frac{V_{\mathrm{u}}}{V_{2}} \phi_{2}+\left(\frac{V_{\mathrm{u}}}{V_{1}} \phi_{1} \chi_{13}+\frac{V_{\mathrm{u}}}{V_{2}} \phi_{2} \chi_{23}\right) \\
\times\left(\phi_{1}+\phi_{2}\right)-\frac{V_{\mathrm{u}}}{V_{1}} \phi_{1} \phi_{2} \chi_{12}-\frac{V_{\mathrm{u}}}{V_{1}} \phi_{1} \phi_{2} \phi_{3} \frac{\partial \chi_{13}}{\partial \phi_{2}} \\
-\frac{V_{\mathrm{u}}}{V_{2}} \phi_{2}^{2} \phi_{3} \frac{\partial \chi_{23}}{\partial \phi_{2}}+\left(\frac{V_{\mathrm{u}}}{V_{1}} \phi_{1} \frac{\partial \chi_{13}}{\partial \phi_{3}}+\frac{V_{\mathrm{u}}}{V_{2}} \phi_{2} \frac{\partial \chi_{23}}{\partial \phi_{3}}\right) \\
\times \phi_{3}\left(\phi_{1}+\phi_{2}\right)-\frac{V_{\mathrm{u}}}{V_{1}} \phi_{1} \phi_{2}^{2} \phi_{3} \frac{\partial \chi_{\mathrm{T}}}{\partial \phi_{2}}-\frac{V_{\mathrm{u}}}{V_{1}} \phi_{1} \phi_{2} \phi_{3} \\
\times\left(\phi_{1}+\phi_{3}\right) \frac{\partial \chi_{\mathrm{T}}}{\partial \phi_{3}}+\frac{V_{\mathrm{u}}}{V_{1}} \chi_{\mathrm{T}} \phi_{1} \phi_{2}\left(1-2 \phi_{3}\right)=0
\end{gathered}
$$

where $V_{\mathrm{u}}$ is the volume of polymer per mole repeating unit. The heat of fusion of polymer $\left(\Delta H_{\mathrm{f}}\right)$ at $25^{\circ} \mathrm{C}$ was determined by differential scanning calorimetry (DSC).

Because the volume fraction of each component adds up to unity in each phase, Eqs. (2)-(6) by using interaction parameters can be calculated to give the phase behavior of PVA in water-DMSO mixtures. Both the experimental liquid-liquid phase equilibrium and the crystallization equilibrium were used to obtain the best fitted phase behavior. The concentration-dependent interaction parameter for water-DMSO binary pair, $\chi_{12}$, was obtained using vapor liquid equilibrium data [18]. The details of calculation for $\chi_{12}$ can be found in [7]. At $25^{\circ} \mathrm{C}$, the osmotic pressure of PVA in solvent was measured and used to calculate the interaction parameters between polymer and solvent, $\chi_{13}$ and $\chi_{23}$, which were simply 
considered as concentration independent [11]. However, there has been no experimental data quantitative analysis for the ternary interaction parameter, $\chi_{\mathrm{T}}$, in the extensive composition range as far as we know. In this study, we treated $\chi_{\mathrm{T}}$ as an empirical correction parameter. For the details of the computational scheme, one can refer to [16].

\section{Experimental}

\subsection{Material}

The PVA with average molecular weight of 74,800 and a degree of saponification of $99.2 \mathrm{~mol} \%$ was kindly supplied by Chang Chun Co. Ltd., Taiwan. ACS-grade DMSO (TEDIA Chem., USA) was used as received. Water was deionized and double distilled before use.

\subsection{Methods: liquid-liquid demixing and crystallization-induced gelation boundaries}

The liquid-liquid demixing and crystallizationinduced gelation boundaries were determined by a cloud point method described in a previous publication [7]. A specific amount of dried PVA polymer was mixed with a suitable amount of water-DMSO mixture and sealed in a Teflon-lined bottle. This mixture was mechanically agitated at $90^{\circ} \mathrm{C}$ until a clear homogeneous solution was obtained. The solution was then maintained at $25^{\circ} \mathrm{C}$ to examine the phase behavior. Due to the time required for gelation in this system depending on a lot of factors such as the PVA concentration, temperature, and the proportion of water and DMSO [2], the phase diagram was established until no further change of phase behavior was observed. The longest observation time was about 1 year.

With different composition, three types of phaseseparated results were observed: (i) solution precipitated into a translucent or a white solid gel; (ii) solution underwent liquid-liquid phase separation into two clear liquid phases; (iii) following case (ii), solution then became a clear liquid phase coexisting with a white solid. The equilibrium gelation point (case (i)) was identified as the composition at which a sharp increase in turbidity was observed by a turbidity bridge (Digital Turbidimeter, Orbeco-Hellige, USA). For case (ii), the location of binodal in the ternary phase diagram was determined and no other phase transition interfered with the liquid-liquid demixing process. For case (iii), gelation occurred in the polymer-rich region after the liquid-liquid demixing. Therefore, the location of the liquid-liquid miscibility gap and gelation region overlapped in the phase diagram.

\subsection{Osmotic pressure measurements}

Osmosis is the passage of a pure solvent into a solution, which is separated by a semi-permeable membrane. The dilute PVA solution was injected into an osmometer (Knauer, Berlin) to determine the osmotic pressure at $25^{\circ} \mathrm{C}$. Then the osmotic pressure of PVA solution was used to calculate the interaction parameters between polymer and solvent, $\chi_{13}$ and $\chi_{23}$. The following equation was employed:

$$
\begin{aligned}
& \frac{\pi}{R T C_{\mathrm{p}}}=\frac{1}{M_{\mathrm{p}}}+\left[\left(\rho_{\mathrm{p}}\right)^{2} V_{i}\right]^{-1}\left(\frac{1}{2}-\chi_{i 3}\right) C_{\mathrm{p}} \\
& i=1 \text { or } 2
\end{aligned}
$$

where $\pi$ is the osmotic pressure, $C_{\mathrm{p}}$ the mass of polymer per unit volume of the solution, $M_{\mathrm{p}}$ the molecular weight of polymer, $\rho_{\mathrm{p}}$ the density of the polymer. By making measurements at several concentrations, extrapolating to zero concentration, the binary interaction parameter between the solvent and polymer was established from the initial slope of a plot of $\pi / R T C_{\mathrm{p}}$ versus $C_{\mathrm{p}}[11]$.

\subsection{DSC analysis}

The thermal property of the PVA gel was carried out using a DSC 2010 (TA Instruments Ltd., USA), calibrated using indium as a standard. The temperature was raised from $4^{\circ} \mathrm{C}$ to the melting point of the gel at a heating rate of $5^{\circ} \mathrm{C} / \mathrm{min}$ to observe the endothermic peak of calorimetric transition around $25^{\circ} \mathrm{C}$.

\section{Results and discussion}

\subsection{Experimental phase behavior}

The phase diagram of water-DMSO-PVA at $25^{\circ} \mathrm{C}$ is shown in Fig. 2 and compared to the system of 
water-DMSO-EVAL constructed in our previous report [7]. The data points, denoted by filled triangles and circles, represent the compositions of the binodal and crystallization-induced gelation boundaries, respectively. In the water-DMSO-EVAL system (Fig. 1), the crystallization-induced gelation and binodal boundaries divide the phase diagram into three parts. Any solution close to the solvent apex on the left of the gelation boundary is single-phase and homogeneous. On the other hand, the binodal boundary is located inside the gelation region. Any composition between the gelation and the binodal boundaries will undergo solid-liquid demixing to form a gel that is crystalline EVAL dispersing uniformly in the liquid [7]. The region close to the nonsolvent apex on the right of the binodal boundary is metastable with respect to both liquid-liquid demixing and crystallization. In contrast to Fig. 1, the water-DMSO-PVA system in Fig. 2 is more complicated. There are two binodal boundaries and two crystallization-induced gelation boundaries. PVA is dissolved in DMSO and water individually at low polymer concentrations, but an originally homogeneous solution becomes gelled or separates into a polymer-rich phase in equilibrium with a polymer-poor solution in the water-DMSO mixture. It is known that gelation rate based on crystallization is connected with a lot of factors such as the polymer concentration and temperature. Actually, the observation time for this study was about 1 year. After 1 year, the phase boundary almost did not show any variation, i.e. phase equilibrium was practically established. Such a phase diagram seems to represent two symmetrical polymer-nonsolvent-solvent systems in the one figure; one is on the left part and the other on the right part of the phase diagram. Therefore, water and DMSO play the roles of nonsolvent and solvent simultaneously in this system. Furthermore, as the polymer content exceeded about $20 \%$, the experimental phase-equilibrium data could not be measured because a uniform solution could not be prepared. Hence, calculations of phase equilibrium were performed to predict a complete phase diagram.

\subsection{Calculated phase behavior}

In Fig. 3, calculated binodals with a constant ternary interaction parameter $\chi_{\mathrm{T}}$ determined by solving Eqs. (2)-(5) and the experimental liquid-liquid

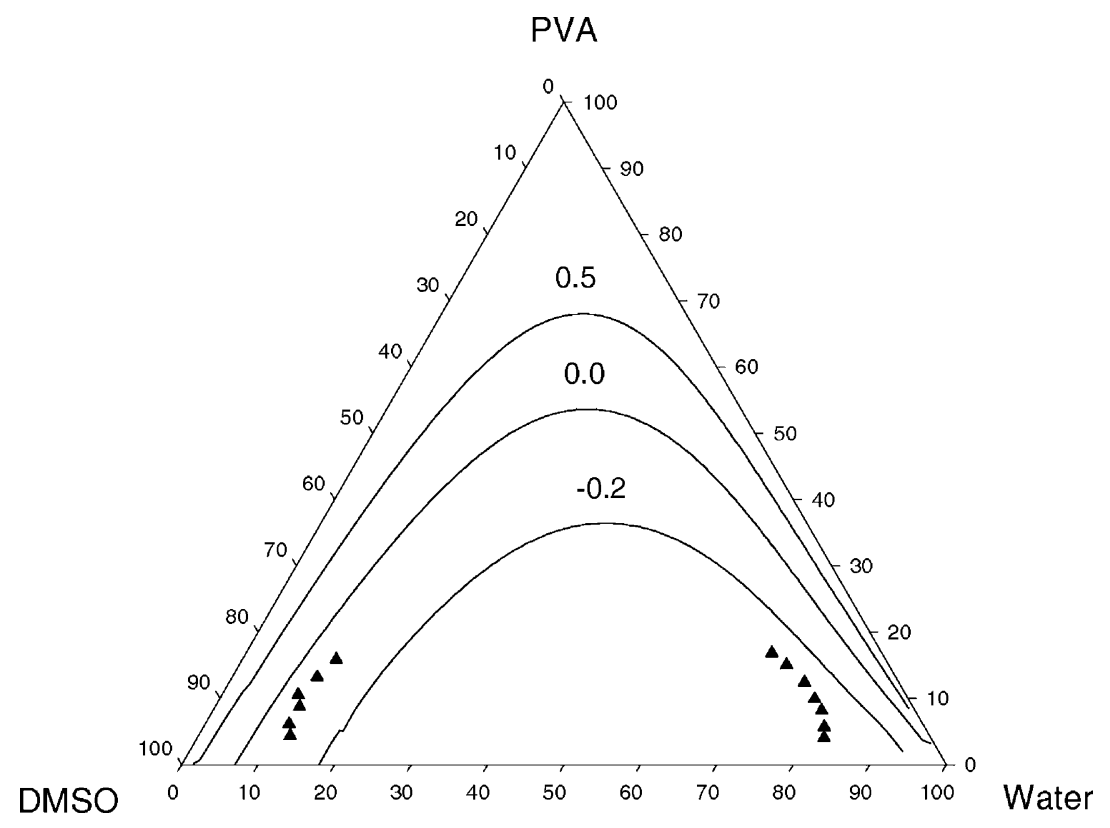

Fig. 3. Comparison between theoretically calculated binodals and experimentally determined binodal boundary data points (denoted by filled triangular symbols $(\boldsymbol{\Delta})$ ). Solid lines represent calculated binodal boundaries for $\chi_{\mathrm{T}}=0.5,0$ and -0.2 . Numbers indicate the value of $\chi_{\mathrm{T}}$ used for the calculations. 
Table 1

Physical constants for PVA polymer

\begin{tabular}{lll}
\hline$T_{\mathrm{m}}\left({ }^{\circ} \mathrm{C}\right)$ & $\rho\left(\mathrm{g} / \mathrm{cm}^{3}\right)$ & $M(\mathrm{~g} / \mathrm{mol})$ \\
\hline 226.7 & 1.26 & 74,800
\end{tabular}

$T_{\mathrm{m}}$, melting temperature; $\rho$, density; $M$, molecular weight.

demixing data points are shown. The physical constants for PVA employed in computations are given in Table 1. The concentration-dependent interaction parameter $\chi_{12}$ (water-DMSO) was determined by using vapor liquid equilibrium data [18] and the results are given in Table 2 . The values of $\chi_{13}$ and $\chi_{23}$ were found to be 0.509 and 0.328 , respectively, from osmotic pressure experiments of polymer solution at $25^{\circ} \mathrm{C}$. As expected, these two values are nearly consistent with both water and DMSO being solvents for PVA.

The value of $\chi_{\mathrm{T}}$ was zero for the first analysis that means the ternary interaction parameter was neglected. Compared to the experimental data, a larger liquid-liquid demixing region was predicted. This means only binary interaction parameters could not be used to properly describe the liquid-liquid phase-equilibrium boundary. Thus, it was further attempted to see if it would be possible to fit the experimental data by considering the ternary interaction parameter. When $\chi_{\mathrm{T}}=0.5$ was considered, the calculated liquid-liquid phase-equilibrium boundary completely disagreed with the experimental data (Fig. 3), in which the liquid-liquid demixing region was enlarged to more near the polymer apex. A positive value for $\chi_{\mathrm{T}}$ was thought to have caused this poorer agreement with the experimental data points. Thus, there ought to be some specific interactions among the three components to decrease the calculated liquid-liquid demixing region. A negative value for $\chi_{\mathrm{T}}$ may account for the effect of an interaction among the three components. Clearly, the calculation

Table 2

Summary of interaction parameter data at $25^{\circ} \mathrm{C}$

\begin{tabular}{lc}
\hline Binary systems & Interaction parameter, $\chi_{i j}$ \\
\hline Water (1)-DMSO (2) & $-1.302+1.08 /\left(1.0+0.86 \phi_{2}\right)$ \\
Water (1)-PVA (3) & 0.509 \\
DMSO (2)-PVA (3) & 0.328
\end{tabular}

$\overline{\phi_{i}}$, volume fraction of component $i(i=1$ : water; $i=2$ : DMSO; $i=3$ : PVA). for $\chi_{\mathrm{T}}=-0.2$ predicted a liquid-liquid demixing region was decreased. On the other hand, the experimental gelation data was also compared to the crystallization equilibrium line determined by solving Eq. (6) (see Fig. 4). The heat of fusion of the PVA gel $\left(\Delta H_{\mathrm{f}}\right)$ at $25^{\circ} \mathrm{C}$ was about $4.093 \mathrm{~J} / \mathrm{g}$ obtained from the DSC analysis as shown in Fig. 5. Likewise, no improvement was obtained for fitting the experimental gelation data in the DMSO-rich region for $\chi_{\mathrm{T}}>0$ (Fig. 4). However, for $\chi_{\mathrm{T}}=-0.2$, a little better fit with experimental data was obtained. It is clear that the experimentally found trends were appropriately predicted between $\chi_{\mathrm{T}}=-0.2$ and 0.0 . Furthermore, for polymer concentrations of less than about $14 \%$, the homogeneous solution was observed at water concentrations of larger than about $87 \%$, whereas the theoretical prediction was not found. The paradox between the theoretical prediction and the experimental gelation data in the water-rich region will be discussed later.

Based on the above result, $\chi_{\mathrm{T}}$ can enlarge or decrease the binodal envelope greatly. In addition, as shown in Fig. 3, the positions of the calculated binodal envelop for $\chi_{\mathrm{T}}=-0.2$ relative to the experimental data are lower in the DMSO-rich region, but higher in the water-rich region. Thus, it was further attempted to see if it would be possible to fit the experimental data by using a concentration-dependent $\chi_{\mathrm{T}}$. In this study, a three-parameter rational form of $\chi_{\mathrm{T}}$ made by Young et al. [16] was followed.

$\chi_{\mathrm{T}}=a+b \phi_{2}+c \phi_{3}$

A trial-and-error procedure was used to obtain the concentration-dependent $\chi_{\mathrm{T}}$. Fig. 6 shows that the calculated binodal boundary by using $\chi_{\mathrm{T}}=$ $-0.28+0.18 \phi_{2}+0.12 \phi_{3}$ matches closely the experimental data points, suggesting that a reliable curve fitting has been obtained. Thus, this concentration-dependent $\chi_{\mathrm{T}}$ was then used with Eq. (6) to calculate the crystallization-induced gelation boundary. Fig. 6 shows that in this case the computed gelation boundary agrees well with experimental data points in the DMSO-rich region of the phase diagram. However, the calculation still predicted the PVA crystallization over the whole right part of the ternary phase diagram.

As described in the Introduction, water does not exist within the PVA as freezable water until the 


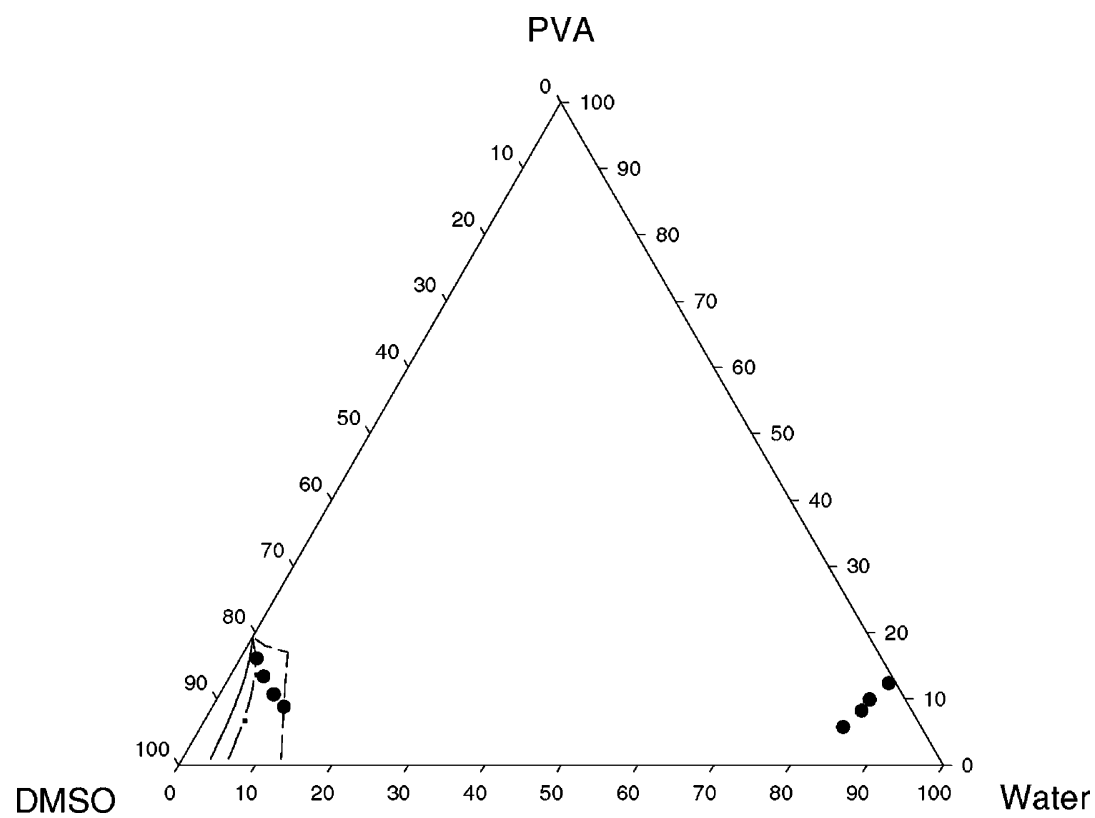

Fig. 4. Comparison between theoretically calculated crystallization equilibrium curves and measured crystallization-induced gelation boundary data points (denoted by filled circles $(-)$ ). Calculated crystallization equilibrium lines for different value of $\chi_{\mathrm{T}}:(-) \chi_{\mathrm{T}}=0.2$; $(-\cdot) \chi_{\mathrm{T}}=0.0 ;$ and $(--) \chi_{\mathrm{T}}=-0.2$.

hydrogen bonding in the PVA has been disrupted by the nonfreezing and freezable bound water. Therefore, in the water-rich region, water dissolves the crystallites of PVA polymer to tend towards completely

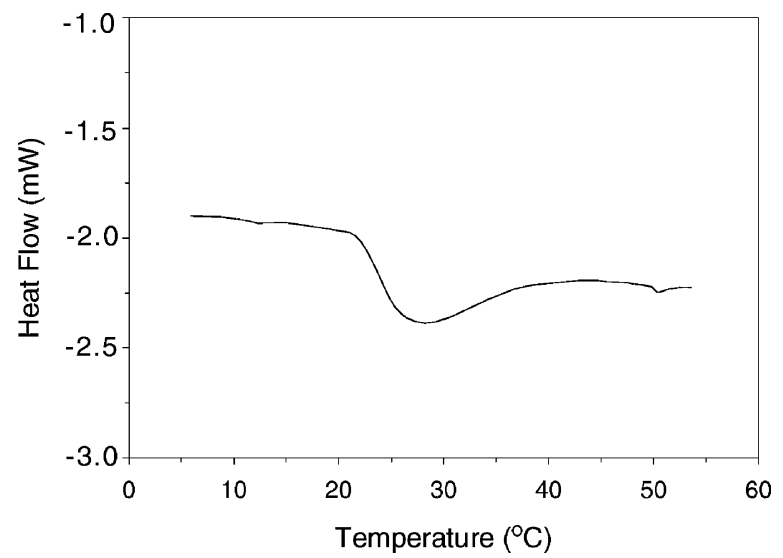

Fig. 5. DSC thermograms of crystalline PVA gel from $4{ }^{\circ} \mathrm{C}$ to the melting points of the gel at a rate of $5^{\circ} \mathrm{C} / \mathrm{min}$. The melting point, $T_{\mathrm{m}}=27^{\circ} \mathrm{C}$. Enthalpy of fusion, $\Delta H_{\mathrm{f}}=4.093 \mathrm{~J} / \mathrm{g}$. The enthalpy data was utilized to compute the equilibrium crystalline line by solving Eq. (6). amorphous content and the crystallization-induced PVA gelation cannot be formed. This is consistent with the results reported from wide-angle X-ray and DSC investigations that diffusion of water into the crystalline fractions in PVA gradually destroys crystallinity in PVA [13]. On the other hand, water actually cannot dissolve PVA polymer at $25^{\circ} \mathrm{C}$ (in this study, PVA polymer was dissolved in water at $90^{\circ} \mathrm{C}$ ). Conversely, PVA polymer could not crystallize from aqueous solution at $25^{\circ} \mathrm{C}$. We use the idea of the activation energy, the barrier that the contacting molecules must surmount if they are to attract rather than withdraw from one another, in the relation between PVA and water. The hydrophilic portion of PVA is embedded into the interior due to the intramolecular hydrogen bonding and PVA offers a hydrophobic surface to water molecules adjacent to it. Likewise, water molecules will group together so that a number of hydrogen bonds are formed in the interior, in which water molecules undergo a hydrophobic hydration to enhance overall structural order. That is to say if PVA and water molecules do not have sufficient thermal energy the redistribution of intramolecular hydrogen bonding of PVA will 


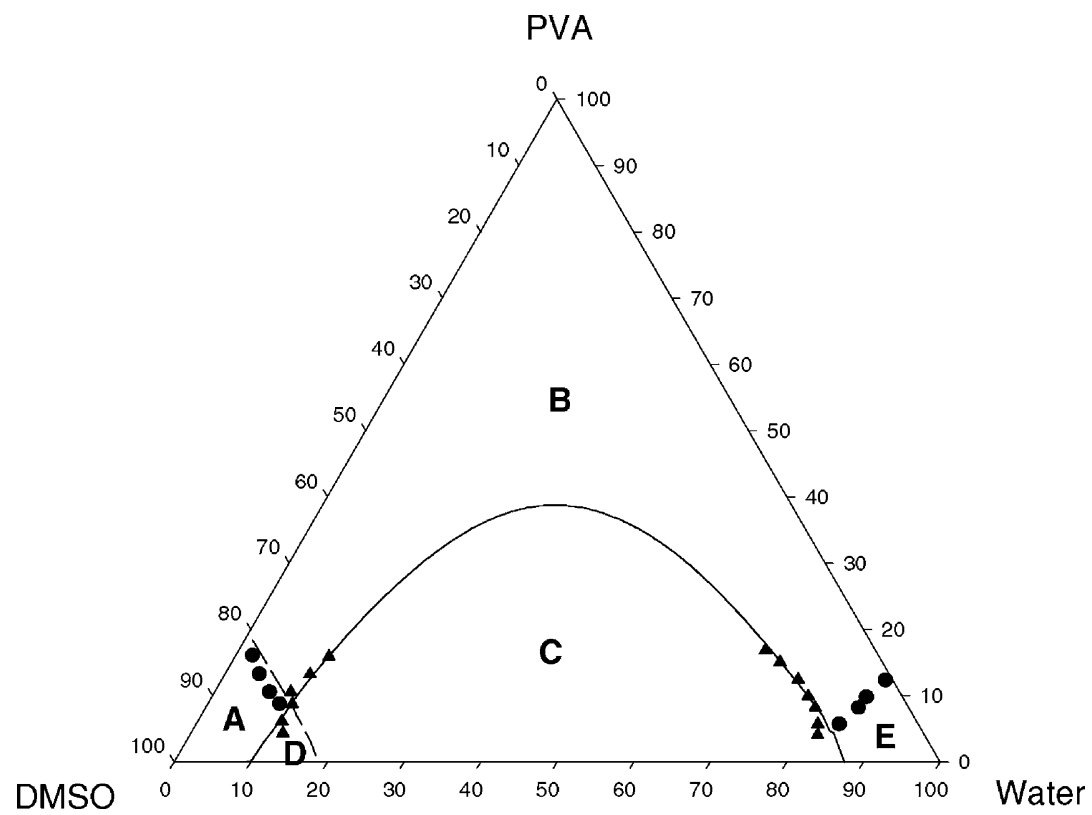

Fig. 6. Comparison between theoretically calculated phase behavior using $\chi_{\mathrm{T}}=-0.28+0.18 \phi_{2}+0.12 \phi_{3}$ and experimentally determined phase transition: $(\mathbf{O})$ measured crystallization-induced gelation boundary data points; $(\mathbf{\Delta})$ measured binodal boundary data points; $(-)$ computed binodal; (--) computed crystallization equilibrium line.

never occur in such a manner that water will recoil from the PVA molecule and no PVA dissolution will take place. When the temperature is increased, the dissociation of intramolecular hydrogen bonding in the PVA becomes possible, resulting in the formation of intermolecular hydrogen bonding between PVA and nonfreezing water in which water drives PVA dissociation. Conversely, the intermolecular hydrogen bonding between PVA and nonfreezing water will impede PVA crystallization from water at $25^{\circ} \mathrm{C}$, that is, PVA would not crystallize from water unless the temperature is increased or some active species (e.g. DMSO) are added to the PVA solution to interact with water to destroy intermolecular hydrogen bonding between PVA and nonfreezing water. Consequently, the irreversible phenomenon of PVA dissolution and crystallization is ascribed to temperature-induced changes in the relative interaction between water and PVA.

The above discussion indicates that the destruction of PVA crystalline structure by nonfreezing water cannot be neglected when the thermodynamic properties of the system water-DMSO-PVA are described by the Flory-Huggins theory. To our knowledge the effect of the water state within the PVA on the interaction parameter between water and PVA has not been studied, presumably because the water state does not usually create problems in the actual conditions. It must be noted, however, that the effect of water state within the polymer on the interaction parameter may become amenable to study, perhaps proving to be significant.

Overall, the complete phase diagram of waterDMSO-PVA that could not be obtained experimentally alone or theoretically alone was constructed from the limited experimental data and Flory-Huggins formalism including three binary interaction parameters and one ternary interaction parameter. Fig. 6 shows the binodal and crystallization-induced gelation boundaries divide the phase diagram into five regions (A, B, C, D and E). Any solution in the region $\mathrm{A}$ is single-phase and homogeneous. The state of solution in region $\mathrm{E}$ is similar to that in region A, but it could be observed experimentally only. Besides in regions $\mathrm{A}$ and $\mathrm{E}$, a sequential development of liquid-liquid phase separation and crystallization will take place for the final gel formation. The region $\mathrm{B}$, defined above binodal and crystallization-induced 
gelation boundaries, is metastable with respect to pure PVA. Any composition in this region will precipitate into a gel induced by crystallization. The regions $\mathrm{C}$, within the binodal phase envelope, are metastable with respect to both liquid-liquid phase separation and crystallization. Actually, Fig. 6 represents two phase diagrams, one approximates true equilibrium between PVA solution and PVA crystals and the other represents liquid-liquid demixing of the super-cooled liquid. Hence, these two phase transitions that are drawn together in Fig. 6 for simplicity cannot really exist together [16,19]. In addition, pure liquid-liquid demixing was observed at region D. This suggests that the location of binodal in this region could be observed experimentally and no other phase transition interfered with the liquid-liquid demixing process.

\subsection{The role of the ternary interaction parameter $\chi_{T}$}

The role of concentration-dependent $\chi_{\mathrm{T}}$ in the water (nonsolvent)-2-propanol (nonsolvent)-EVAL at $60^{\circ} \mathrm{C}$ has been investigated in our previous publication [16]. The optimum $\chi_{\mathrm{T}}$ value was found to be approximately $-1.7+0.5 \phi_{2}+1.0 \phi_{3}$. Such negative values of $\chi_{\mathrm{T}}$ over the all compositions imply a strong interaction among the three components, which even may overweigh binary repulsive interactions. It has been shown that in the Flory-Huggins theory with inserted $\chi_{\mathrm{T}}$ results in the dissolution of EVAL in the water-2-propanol cosolvent [16]. In the present study, if the binodal boundary is considered only the immiscibility characteristic of cononsolvency indeed appear in the phase diagram, in which the solution region encloses a liquid-liquid demixing region near the water-DMSO axis. It seems reasonable to suggest the use of the ternary interaction parameter accounts for the immiscible effect of the solvent pair on a polymer in a cononsolvent system. Thus, a positive $\chi_{\mathrm{T}}$ value is proposed to dominate the repulsive interaction among the three components. However, the $\chi_{T}$ value for the system under study is still negative. Therefore, it is not appropriate to attribute the cononsolvency of PVA in water-DMSO mixtures to the negative $\chi_{\mathrm{T}}$ value.

It is interesting to investigate why does cononsolvency occur in the water-DMSO-PVA system. A series of calculations of ternary polymer systems have been investigated by Altena and Smolders by using
Flory-Huggins formalism with three binary interaction parameters [20]. A larger demixing gap existed for decreasing $\chi_{12}$. For the system under study, the calculated binodal for $\chi_{\mathrm{T}}=0$ was larger than the measured data, as shown in Fig. 3. Thus, the larger calculated binodal can be reasoned by the fact that the estimated $\chi_{12}$ value appears to be too negative. In addition, Wolf and Willms proposed that cononsolvency occurs when the two solvents are close to demixing $\left(\chi_{12} \approx+2\right)$ or when they complex between themselves $\left(\chi_{12}<0\right)$ [8]. Our calculations thus seem to support the preference of water to complex with DMSO rather than with PVA as reflected in the negative value of $\chi_{12}$. Since water-DMSO contacts are favored over water-PVA or DMSO-PVA contacts, PVA molecules in the solution are clustered together to exclude solvent by the addition of another solvent. A similar result has been reported by Katayama et al. [9] that cononsolvency occurs upon addition of DMSO to aqueous poly ( $N$-isopropylacrylamide). Thus, we suggest that the negative $\chi_{12}$ value is the origin of cononsolvency in the water-DMSO-PVA system. On the other hand, the negative $\chi_{\mathrm{T}}$ value favors the cosolvency, not the cononsolvency, which can reduce the demixing region.

\section{Conclusion}

The phase behavior of PVA in water-DMSO mixtures was studied at $25^{\circ} \mathrm{C}$. PVA exhibited cononsolvency in water-DMSO mixtures. Flory-Huggins ternary solution theory could explain the observed results in terms of the solvent-solvent interaction parameter and the ternary interaction parameter. The optimum value of $\chi_{\mathrm{T}}$ was $-0.28+0.18 \phi_{2}+0.12 \phi_{3}$. The very negative $\chi_{\mathrm{T}}$ value indicates this system favoring the formation of water-DMSO-PVA contacts. Thus, the driving force for cononsolvency results from that the water-DMSO complexes are preferred to PVA-water and PVA-DMSO complexes.

\section{Acknowledgements}

The authors thank the National Science Council of Taiwan, the Republic of China for their financial support of this research. 


\section{References}

[1] I. Sakurada, Polyvinyl Alcohol Fibers, Marcel Dekker, New York, 1985.

[2] W.I. Cha, S.H. Hyon, Y. Ikada, Gel spinning of poly (vinyl alcohol) from dimethyl sulfoxide/water mixture, J. Polym. Sci., Polym. Phys. 32 (1994) 297-304.

[3] M. Ohkura, T. Kanaya, K. Kaji, Gels of poly (vinyl alcohol) from dimethyl sulphoxide/water solutions, Polymer 33 (1992) 3686-3690.

[4] T. Kanaya, M. Ohkura, K. Kaji, M. Furusaka, M. Misawa, Structure of poly (vinyl alcohol) gels studied by wide- and small-angle neutron scattering, Macromolecules 27 (1994) 5609-5615.

[5] K. Yamaura, K. Karasawa, T. Tanigami, S. Matsuzawa, Gels of poly (vinyl alcohol) solutions at low temperatures (20 to $-78{ }^{\circ} \mathrm{C}$ ) and properties of gels, J. Appl. Polym. Sci. 51 (1994) 2041-2046.

[6] D.H. Rasmussen, A.P. MacKenzie, Phase diagram for the system water-dimethylsulphoxide, Nature 220 (1968) 13151317.

[7] T.H. Young, J.Y. Lai, W.M. You, L.P. Cheng, Equilibrium phase behavior of the membrane forming water-DMSOEVAL copolymer system, J. Membr. Sci. 128 (1997) 55-65.

[8] B.A. Wolf, M.M. Willms, Measured and calculated solubility of polymers in mixed solvents: co-nonsolvency, Makromol. Chem. 179 (1978) 2265-2777.

[9] S. Katayama, Y. Hirokawa, T. Tanaka, Reentrant phase transition in acrylamide-derivative copolymer gels, Macromolecules 17 (1984) 2641-2643.

[10] H.G. Schild, M. Muthukumar, D.A. Tirrell, Cononsolvency in mixed aqueous solutions of poly ( $n$-isopropylacrylamide), Macromolecules 24 (1991) 948-952.
[11] P.J. Flory, Principles of Polymer Chemistry, Cornell University Press, Ithaca, NY, 1953.

[12] J. Pouchly, A. Zivny, K. Solc, Thermodynamics equilibrium in the system macromolecular coil-binary solvent, J. Polym. Sci. C 23 (1968) 245-256.

[13] R.M. Hodge, G.H. Edward, G.P. Simon, Water absorption and states of water in semicrystalline poly (vinyl alcohol), Polymer 37 (1996) 1371-1376.

[14] A. Higuchi, T. Iijima, DSC investigation of the states of water in poly (vinyl alcohol) membranes, Polymer 26 (1985) 1207-1211.

[15] Y.L. Guan, L. Shao, K.D. Yao, A study on correlation between water state and swelling kinetics of chitosan-based hydrogels, T. Appl. Polym. Sci. 61 (1996) 2325-2335.

[16] T.H. Young, L.P. Cheng, C.C. Hsieh, L.W. Chen, Phase behavior of EVAL polymers in water-2-propanol cosolvent, Macromolecules 31 (1998) 1229-1235.

[17] L.P. Cheng, A.W. Dwan, C.C. Gryte, Isothermal phase behavior of nylon-6, -66, and -610 polyamides in formic acid-water systems, J. Polym. Sci., Polym. Phys. 32 (1994) 1183-1190.

[18] S.Y. Lam, R.L. Benoit, Some thermodynamic properties of the dimethylsulfoxide-water and propylene carbonate-water systems at $25{ }^{\circ} \mathrm{C}$, Can. J. Chem. 52 (1974) 718722.

[19] J.H. Aubert, Isotatic polystyrene phase diagrams and physical gelation, Macromolecules 21 (1988) 3468-3473.

[20] F.W. Altena, C.A. Smolders, Calculation of liquid-liquid phase separation in a ternary system of a polymer in a mixture of a solvent and a nonsolvent, Macromolecules 15 (1982) 1491-1497. 